4526

\section{Coping with End of Life Care: Compassion Fatigue and Burnout Effects on Cortisol Patterns of Health Care Providers Caring for Children \\ Liza Carolina Sanchez-Plazas ${ }^{1}$, Ricardo L. Garcia ${ }^{1}$, and Kelly Komatz $^{2}$ \\ ${ }^{1}$ University of Puerto Rico, Medical Science Campus; ${ }^{2}$ University of Florida, Jacksonville}

OBJECTIVES/GOALS: The objectives are to assess the impact of cumulative grief on the development of Compassion Fatigue (CF) and Burnout Syndrome (BS) in HCPs who care for dying children. We will also evaluate the relationship between $\mathrm{CF}$ and cortisol patterns in HCPs. METHODS/STUDY POPULATION: Cross-sectional study to be conducted in a Pediatric Hospital in Puerto Rico. A sample of 50 pediatric nurses will be selected to collect the data to evaluate the occurrence of $\mathrm{CF}$ and $\mathrm{BS}$ among $\mathrm{HCP}$ caring for children during end of life (EoL). Study subjects will include nurses who care for dying children in the Intensive Care Units and Oncology ward. Nurses working in the pediatric ward will be included as the control group. Three validated instruments (Spanish Version) will be administered (Professional Quality of Life vIV, Maslach Burnout Inventory- HSS, briefCOPE scale). Cortisol samples in saliva and hair will also be taken to determine levels in these HCPs. RESULTS/ANTICIPATED RESULTS: Our expected outcome is that CF and BS will be more frequent in HCPs caring for children during EoL compared with controls and that EoL nurses will have higher scores on CF scale and more frequent dysregulated cortisol patterns. DISCUSSION/SIGNIFICANCE OF IMPACT: Understanding how $\mathrm{HCP}$ s cope with grief caused by child death and the occurrence of $\mathrm{CF}$ and $\mathrm{BO}$ in our hispanic population allowing us to develop support strategies based on the specific HCPs needs. This knowledge will help improve HCPs' well-being and may diminish the physiologic impact on cortisol.

4047

EEG as a Predictor of Post-Stroke Recovery: A Systematic Review and Meta-Analysis*

Amanda Vatinno ${ }^{1}$, Viswanathan Ramakrishnan, $\mathrm{PhD}^{1}$, Annie Simpson, $\mathrm{PhD}^{1}$, Heather Bonilha, PhD, CCC-SLP1, and $\mathrm{Na}$

Jin Seo, $\mathrm{PhD}^{1}$

${ }^{1}$ Medical University of South Carolina

OBJECTIVES/GOALS: The objective of this study is to perform a systematic review and meta-analysis on the prognostic utility of electroencephalography (EEG) in stroke recovery. METHODS/STUDY POPULATION: A literature search was conducted using three electronic databases, including PubMed, Scopus, and CINAHL. Key search terms were "EEG," "stroke," and "rehabilitation". Only peer-reviewed journal articles published in English that examined the relationship between EEG and a standardized clinical outcome measure(s) at a later time in stroke patients were included. Two independent raters completed data extraction and assessed methodological quality of the studies with the Downs and Black form. A linear meta-regression was performed across subsets of individual studies that utilized a common clinical outcome measure to determine the association between EEG and clinical outcome while adjusting for sample size and study quality. RESULTS/ANTICIPATED RESULTS: 56 papers met the inclusion criteria and were included in the systematic review. The prognostic value of EEG was evidenced at both the acute and chronic stages of stroke. The addition of EEG enhanced prognostic accuracy more than initial clinical assessment scores and/or lesion volume alone. In the metaanalysis, a subset of 10 papers that utilized the National Institutes of Health Stroke Scale (NIHSS) and a subset of 7 papers that utilized the Modified Rankin Scale (MRS) were included. Analysis demonstrated an association between EEG and the subsequent clinical outcome measures. DISCUSSION/SIGNIFICANCE OF IMPACT: Currently, prognosis is largely based on initial behavioral impairment level. However, post-stroke recovery outcomes are heterogeneous despite similar initial clinical presentations. Uncertain prognosis makes it difficult for clinicians to develop personalized treatment plans for patients. Improved prognosis for recovery may guide clinical management for stroke survivors by helping clinicians determine the maximally efficient course of treatment and care. This study suggests that prognostic accuracy may be enhanced using EEG.

4049

Evaluating Miami CTSI's Pilot, Translational, and Clinical Studies Program using research success measures and CTSA Common Metrics

Rosalina Das ${ }^{1}$, Patricia Avissar ${ }^{2}$, Jessica Diaz ${ }^{2}$, Sheela C Dominguez $z^{2}$, Barry S. Issenberg ${ }^{2}$, Dalton W. Dietrich ${ }^{2}$, and Ralph L. Sacco ${ }^{2}$

${ }^{1}$ University of Miami Clinical and Translational Science Institute; ${ }^{2}$ University of Miami

OBJECTIVES/GOALS: The goal of this project was to a) evaluate the first five years of Miami CTSI's Pilot Translational and Clinical Studies Program using outcome measures that quantify research productivity augmented by the CTSA Common Metrics; and b) use the results to shape future program management. METHODS/STUDY POPULATION: Pilot Program applicant and awardee demographic data were collected during the first 5-year cycle of the Miami CTSI grant. Projects were categorized into the translation spectrum based on type of research using published guidelines. Research productivity from funded pilot projects were tracked annually using internal institutional grant award databases and external databases such as PubMed and NIH Reporter. CTSA Common Metrics were tracked using the Results Based Accountability framework. Relative Citation Ratio (RCR), NIH percentile and translation impact of pilot project publications were determined using the iCite tool (NIH Office of Portfolio Analysis). RESULTS/ANTICIPATED RESULTS: The Miami CTSI's Pilot Award Program demonstrated notable success in its first five years. Of the twenty-two projects that were funded during that time period, $45 \%$ led to follow-on funding for a total of $\$ 17.2 \mathrm{M}-$ a strong return on investment of 15:1. Further, 77\% of awardees had at least one publication. A total of four patents and 43 publications resulted directly from the funded projects. The mean RCR for all publications was 2.7, weighted RCR was 99.87 , and nine papers were been cited by clinical documents. Overall, $63 \%$ of the projects were classified as $\mathrm{T} 1 / \mathrm{T} 2$ (pre-clinical/clinical research) and 37\% as T3/T4 (post-clinical translational research/public health). DISCUSSION/SIGNIFICANCE OF IMPACT: Miami CTSI's Pilot Award Program demonstrated success in scholarly output, follow on funding, and scientific impact. These results will serve as benchmarks going forward and will allow the CTSI to leverage program strengths in collaborating with other institutional internal award mechanisms. 